\title{
AnyPyTools: A Python package for reproducible research with the AnyBody Modeling System
}

\author{
Morten Enemark Lund ${ }^{1,2}$, John Rasmussen², and Michael Skipper \\ Andersen $^{2}$
}

1 AnyBody Technology A/S, Denmark 2 Department of Materials and Production, Aalborg University, Denmark

DOI: $10.21105 /$ joss.01108

\section{Software}

- Review ¿

- Repository ${ }^{\top}$

- Archive ${ }^{\top}$

Submitted: 26 November 2018 Published: 07 January 2019

\section{License}

Authors of papers retain copyright and release the work under a Creative Commons Attribution 4.0 International License (CC-BY).

\section{Summary}

The AnyPyTools package provides a Python interface to automate multibody musculoskeletal model simulations in the AnyBody Modeling System. The main advantage of AnyPyTools is that it enables reproducible research for the AnyBody Modeling System, and bridges the gap to the whole ecosystem of open source scientific Python packages.

As musculoskeletal simulations become increasingly important in decision making processes in a range of applications, so does the requirement for model verification and validation (V\&V) (M. E. Lund, Zee, Andersen, \& Rasmussen, 2012). Successful V\&V will often require running large numbers of simulations (batch processing) or investigating parameters systematically (sensitivity or parameter studies). The stand-alone AnyBody Modeling System is not very suited for this kind of meta-analysis. The modeling system is essentially an IDE/compiler for scripting single multibody musculoskeletal models in the AnyScript modeling language.

The AnyPyTools Python package enables batch processing, parallelization of model simulations, model sensitivity studies, and parameter studies, using either Monte-Carlo (random sampling) or Latin hypercube sampling. It makes reproducible research much easier and replaces the tedious process of manually automating the musculoskeletal simulations and aggregating the results.

The AnyPyTools library was developed at Aalborg University to help in the effort to validate musculoskeletal models created within the AnyBody Modeling System (M. E. Lund, Andersen, Zee, \& Rasmussen, 2015, M. E. Lund et al. (2012)). In this work AnyPyTools was used to orchestrate large number of model simulations and distribute the load over multiple processors, as well as collect the results directly in Python and investigate the sensitivity of the model predictions. The library has evolved over time to also include a pytest plugin for running unit tests on AnyScript files (test_*.any) similar to how unit-tests are used for Python.

The AnyPyTools library is available on both PyPI and conda. It has been downloaded more than 20.000 times from the conda-forge channel and has been used in a large number of scientific publications over the last 5 years (De Pieri et al., 2018, Stensgaard Stoltze, Rasmussen, \& Skipper Andersen (2018), Richards, Andersen, Harlaar, \& Noort (2018), Theodorakos et al. (2018), Rasmussen2018-nq, Dell'Isola, Smith, Andersen, \& Steultjens (2017), Eltoukhy, Kuenze, Andersen, Oh, \& Signorile (2017), Skals, Rasmussen, Bendtsen, Yang, \& Andersen (2017), Anderson Souza Oliveira, Silva, Lund, Farina, \& Kersting (2017), Skipper Andersen, Zee, Damsgaard, Nolte, \& Rasmussen (2017), Skals, Jung, Damsgaard, \& Andersen (2017), Vanheule et al. (2017), Theodorakos et al. (2016), M. E. 
Lund et al. (2015), A. S. C. Oliveira, Silva, Lund, Farina, \& Kersting (2014), Anderson Souza Oliveira et al. (2013), A S Oliveira, Silva, Lund, Kersting, \& Farina (2013))

The source code for AnyPyTools is available on GitHub and releases are archived to Zenodo with the linked DOI: (M. E. Lund, Andersen, \& Rasmussen, 2018)

\section{Acknowledgements}

We acknowledge contributions from AnyBody Technology A/S who have used the package extensively for their verification and validation work. Also, thanks to to the numerous academic users of the AnyBody Modeling System from all over the world, who have contributed feedback and feature requests.

\section{References}

De Pieri, E., Lund, M. E., Gopalakrishnan, A., Rasmussen, K. P., Lunn, D. E., \& Ferguson, S. J. (2018). Refining muscle geometry and wrapping in the TLEM 2 model for improved hip contact force prediction. PLoS One, 13(9), e0204109. doi:10.1371/journal.pone.0204109

Dell'Isola, A., Smith, S. L., Andersen, M. S., \& Steultjens, M. (2017). Knee internal contact force in a varus malaligned phenotype in knee osteoarthritis (KOA). Osteoarthritis Cartilage, 25(12), 2007-2013. doi:10.1016/j.joca.2017.08.010

Eltoukhy, M., Kuenze, C., Andersen, M. S., Oh, J., \& Signorile, J. (2017). Prediction of ground reaction forces for parkinson's disease patients using a kinectdriven musculoskeletal gait analysis model. Med. Eng. Phys., 50, 75-82. doi:10.1016/j.medengphy.2017.10.004

Lund, M. E., Andersen, M. S., \& Rasmussen, J. (2018, December). AnyPyTools. doi:10.5281/zenodo. 1251087

Lund, M. E., Andersen, M. S., Zee, M. de, \& Rasmussen, J. (2015). Scaling of musculoskeletal models from static and dynamic trials. International Biomechanics, 2(1), 1-11. doi:10.1080/23335432.2014.993706

Lund, M. E., Zee, M. de, Andersen, M. S., \& Rasmussen, J. (2012). On validation of multibody musculoskeletal models. Proc. Inst. Mech. Eng. H, 226(2), 82-94.

Oliveira, A. S. C., Silva, P. B., Lund, M. E., Farina, D., \& Kersting, U. G. (2014). Slipping during side-step cutting: Anticipatory effects and familiarization. Hum. Mov. Sci., 34, 128-136. doi:10.1016/j.humov.2013.12.009

Oliveira, A. S., Silva, P. B., Lund, M. E., Farina, D., \& Kersting, U. G. (2017). Balance training enhances motor coordination during a perturbed sidestep cutting task. J. Orthop. Sports Phys. Ther., 47(11), 853-862. doi:10.2519/jospt.2017.6980

Oliveira, A. S., Silva, P. B., Lund, M. E., Gizzi, L., Farina, D., \& Kersting, U. G. (2013). Effects of perturbations to balance on neuromechanics of fast changes in direction during locomotion. PLoS One, 8(3), e59029. doi:10.1371/journal.pone.0059029

Oliveira, A. S., Silva, P. B., Lund, M. E., Kersting, U. G., \& Farina, D. (2013). Fast changes in direction during human locomotion are executed by impulsive activation of motor modules. Neuroscience, 228, 283-293. doi:10.1016/j.neuroscience.2012.10.027

Richards, R. E., Andersen, M. S., Harlaar, J., \& Noort, J. C. van den. (2018). Relationship between knee joint contact forces and external knee joint moments in patients with 
medial knee osteoarthritis: Effects of gait modifications. Osteoarthritis Cartilage, 26(9), 1203-1214. doi:10.1016/j.joca.2018.04.011

Skals, S., Jung, M. K., Damsgaard, M., \& Andersen, M. S. (2017). Prediction of ground reaction forces and moments during sports-related movements. Multibody Syst. Dyn., 39(3), 175-195. doi:10.1007/s11044-016-9537-4

Skals, S., Rasmussen, K. P., Bendtsen, K. M., Yang, J., \& Andersen, M. S. (2017). A musculoskeletal model driven by dual microsoft kinect sensor data. Multibody Syst. Dyn., 41(4), 297-316. doi:10.1007/s11044-017-9573-8

Skipper Andersen, M., Zee, M. de, Damsgaard, M., Nolte, D., \& Rasmussen, J. (2017). Introduction to Force-Dependent kinematics: Theory and application to mandible modeling. J. Biomech. Eng., 139(9). doi:10.1115/1.4037100

Stensgaard Stoltze, J., Rasmussen, J., \& Skipper Andersen, M. (2018). On the biomechanical relationship between applied hip, knee and ankle joint moments and the internal knee compressive forces. International Biomechanics, 1-12. doi: $10.1080 / 23335432.2018 .1499442$

Theodorakos, I., Rueterbories, J., Lund, M. E., Andersen, M. S., Zee, M. de, \& Kersting, U. G. (2016). Ankle bracing effects on knee and hip mechanics during landing on inclined surfaces. International Biomechanics, 3(1), 22-32. doi:10.1080/23335432.2015.1132638

Theodorakos, I., Rueterbories, J., Lund, M. E., Eils, E., Andersen, M. S., De Zee, M., \& Kersting, U. G. (2018). Effects of a semi-rigid ankle brace on ankle joint loading during landing on inclined surfaces. International Biomechanics, 1-11. doi: $10.1080 / 23335432.2018 .1481767$

Vanheule, V., Delport, H. P., Andersen, M. S., Scheys, L., Wirix-Speetjens, R., Jonkers, I., Victor, J., et al. (2017). Evaluation of predicted knee function for component malrotation in total knee arthroplasty. Med. Eng. Phys., 40, 56-64. doi:10.1016/j.medengphy.2016.12.001 